\title{
The 'Coming of Age' of Telehealth, with NCOVID-19 in Context
}

\author{
Babu George, PhD, DBA, EdS \\ Fort Hays State University, USA \\ Email: bpgeorge@fhsu.edu \\ ORCID: https://orcid.org/0000-0002-2791-828X \\ Lena Bucatariu, DBA \\ RMIT Vietnam, Ho Chi Minh City, Vietnam \\ Email: lena.bucatariu@gmail.com \\ ORCID https://orcid.org/0000-0001-6015-9054
}

\begin{abstract}
Telehealth has been playing a progressively major role in the management of the NCOVID-19 crisis. The enforcement of social distancing measures has had the consequence of reduced technology distance in almost every walk of life. In this paper, based primarily on the still unfolding experiences of deploying it during the current situation, we argue that telehealth has finally come of age and that it is time to move it from the peripheries to the center of the 21st century healthcare. To provide a live context to the discussion, several instances of how telehealth strengthened our healthcare systems during the NCOVID-19 crisis are presented.

Keywords: Telehealth, telemedicine, NCOVID-19, technology, management, regulations.

\section{Introduction}

Telehealth has been an active topic in scholarly debates and in the field of medical practice, since the 1980's or even before (Tuckson, Edmunds, \& Hodgkins, 2017). Telemedicine, its subset focusing specifically on the delivery of clinical care, used to be a relatively more popular term (Ohannessian, 2015). Telehealth covers a broader range of technologies and services to the patient, in turn improving the overall healthcare delivery system (Smith et al., 2020).
\end{abstract}


More recently, there have been a lot of interesting developments in technologies, medical profession, and in the State, national, and international legal frameworks guiding the adoption of telehealth (Portnoy, Waller, \& Elliott, 2020). Of particular importance to notice is that telehealth has now assumed greater importance given the suppleness it offers in crisis situations; we see it today, as NCOVID-19 continues to take it tolls (Calton, Abedini, \& Fratkin, 2020; Hollander \& Carr, 2020; Smith et al., 2020). In fact, this current crisis may have potentially raised telehealth to its 'coming of age', from the fringes to the mainstream. It also saw the healthcare practitioners adopting telehealth in truly creative and hitherto unknown ways (Deepak, Subuhi, \& Ishmeet, 2020).

\section{Background}

The telehealth revolution has brought into existence numerous novel kinds of businesses in the broad wellness spectrum (George \& Henthorne, 2009). The general health assessments made possible by these technologies make prognosis and preventive interventions feasible for the masses. Advances in information and communication technologies have transformed almost every device used in healthcare as a part of the globally connected Internet of Things (Dinesen et al., 2016). The coming together of the internet, smart devices, health insurance companies, and Accountable Care Organizations (ACOs) is fundamentally changing the way the physicians practice medicine and also the very business models of healthcare businesses.

Introduction of advanced robotics into healthcare means that not only consultations but sophisticated surgical procedures could also be performed remotely ((Ohannessian, 2015). In the more recent iteration, with the implementation of Electronic Intensive Care Units (eICU), especially in acute care situations, doctors could stay at home and remotely monitor patients admitted in hospitals. The use of big data analytics in telehealth has become more common recently (Wang, Qiu, \& Guo, 2017). Among other things, this helps predict problem populations and patients in advance. This way, healthcare providers could better target treatments for diseases like chronic heart diseases and diabetes. Several public health experts have stressed the need to develop an integrated and globally distributed telehealth system that would thrive on big data sourced from around the world (Mishra \& Chakraborty, 2020). 
For healthcare facilities offering specialized and high-end services at affordable prices, say, those in India, Thailand, Costa Rica, etc., there has been a burgeoning consumer demand for telehealth from across national borders. Typically, everything but invasive procedures are done remotely and then the patients fly to these destinations for hospitalized treatments. Preventive medicine and mental health are prime candidates for telemedicine (Bucatariu \& George, 2017; George, Henthorne, \& Williams, 2010). In this scenario, the medical tourism profession has found a good partner in telehealth as health insurance companies are becoming increasingly open to approving such alternatives as well.

\section{The NCOVID-19 in Context}

By now, it is widely agreed that NCOVID-19, severe respiratory infection caused by a type of coronavirus, has its (yet unknown) origins in Wuhan, China, around December 2019 or earlier. By March 2020, it became a global pandemic; according to various estimates, the average mortality rate is close to $5 \%$. In addition to being a life-threatening situation for many, the exponential proliferation of this disease created havocs in the economic and social fabrics of several countries.

Social distancing measures were advocated globally; telehealth procedures, by their very nature, are a natural partner to the enforcement of these measures in the clinical settings (Ohannessian, Duong, \& Odone, 2020). Countries like Israel and Japan have used telemedicine to provide care to infected passengers stranded on cruise ships for multiple weeks. Infected sailors on the US Navy ships too received extensive telemedicine support. At least ten US navy vessels reported significant outbreaks recently. In China, surprisingly, the government had invested heavily in an Emergency Telemedicine Consultation System (ETCS) and this quickly kicked in to complement the brick and mortar healthcare facilities (Zhai, et al., 2020).

Available anecdotal evidence indicates that telehealth did play a surprisingly stellar job in early diagnosis and the continuous monitoring of symptoms while the patient is held in isolation or quarantine. The consumer directed supply chain leveraged by many hospitals during the NCOVID-19 made it possible for patients to be pre-screened remotely for symptoms and for gathering evidences such as travel history (Zhai et al., 2020). Screening algorithms powered by 
Al did most of these without much of direct human intervention. According to the likelihood scenarios and other constraints, the bots would then intelligently schedule patients to either hospital on-premise facilities or to a live video conference with physicians. Prevention or identifying the symptoms of the disease during early consultations is the most rightful use of telehealth. It does sound like this is the main direction telehealth is currently being used amidst the pandemic (Gao et al., 2020). In developing economies in Asia, telemedicine has come a long way from store-and-forward (Brandling-Bennett et al, 2005) email-based diagnostic in remote rural areas of Cambodia (Heinzelmann et al, 2005) to the development of smart NCOVID-19 helmets with a Mounted Thermal Imaging System that measures temperature in Indonesia (Mohammed et al, 2020), NCOVI software to report symptoms in Vietnam (Nguyen et al, 2020) and Afghanistan's assessment via the Internet of Things (Azizy et al, 2020).

Mobile and e-ICU systems increased the efficiency of the system multifold. A couple of physicians could monitor day and night a hundred or more patients simultaneously, thanks to the electronic distribution system made possible by interconnecting these smart units. If there is a situation involving numerous physicians being quarantined for possible infections, this could be the only workable last resort solution. Although experimentally, some of the hospital systems even used self-driving vans as mobile units. More advancement in these technologies would help lower exposure for others. Even with limited deployment, earlier indications are that telehealth has helped significantly to preserve valuable personal protective equipment.

According to popular media reports, many hospital systems that did not have these technologies and associated infrastructure collaborated with others to benefit from them. Complementary systems such as the ETHAN (Emergency Telehealth and Navigation) being used by the Houston fire department was tweaked to better serve in the current crisis. The seamless coordination of testing centers is vital in maximizing the efficiency of testing. Networking among hospital systems helped further this goal. Yet, true interoperability among these systems was found to be impossible, given the differences in the environments each of them developed. Aurora Health, Cleveland Clinic, Jefferson Health, Kaiser Permanente, Mount Sinai, and Providence, all have their own in-house systems and models and the current crisis points to the need of integrating these systems in a tighter manner. 
The good news is, although these technologies were not being heavily used in the past or not interconnected for the best efficiency, those were available still for relatively quick deployment in the crisis situation (Chauhan et al., 2020). The lack of short and easy to understand training programs such as videos and user guides is something that needs to be quickly addressed.

Training the physicians and practice facility staff is one thing and training the patients and their family is quite a different matter. Agencies like the American Medican Association (AMA), National Consortium of Telehealth Resource Centers (TRCs), and the Centers for Medicare \& Medicaid Services (CMS) has catered well to the former part. Telehealth technology developers too contributed heavily to documenting practice implementation, policy, coding, and payment methodologies. The AMA Physician Innovation Network organized several webinars on telehealth deployment for the benefit of the physicians, too.

Yet, usability of telehealth is not a given thing, especially for the elderly patients (Lor \& George, 2014); the slope of the learning curve needs to be reduced by means of using familiar front-end designs for client end devices and must come with step-by-step guides. Powell et al. (2017) observe that the comfort and familiar home environment in which patients stay while virtually interacting with physicians should make them discuss their conditions more honestly. Obviously, the nature of the illness and any stigma attached to it would moderate this.

Social media technologies could be repurposed to play a central role in the communication function (Menon \& George, 2018). More importantly, in the light of the lowered thresholds of HIPAA compliance as a result of COVID-19, communication tools built around consumeroriented platforms such as Skype, FaceTime, Google Hangouts, etc. could potentially be used for initial consultations. Deepak et al. (2020) narrates how dermatologists in India use WhatsApp for consultations these days. In mental health, the NCOVID-19 pandemic has spun new fields such as telepsychiatry, telepsychology, teletherapy and teleneurology in which social media and other technologies are used to treat patients (Klein et al, 2020). To illustrate, online counselling of hidden youth (i.e. withdrawn from school and social activities) in Hongkong had better therapeutic outcomes than traditional offline methods due to anonymity, fit with lifestyle and more power balance (Chan et al, 2020), although still less effective than integrated delivery. For substance related treatment in the context of NCOVID-19 disruptions, doctors are 
no longer bound by the Drug Enforcement Administration's requirement for face-to-face assessment and prescription, which led to growth in live interactive video communications to assess and medicate ADHD youth (Knopf et al, 2020). A plethora of medical specialties have capitalized on remote treatment, including reports of smartphone based ocular imaging for eye care (Naga et al, 2020), iPads for telepalliative care (Humphreys et al, 2020), virtual management with online instructions for urology infections (Grimes et al, 2020), a portal for pediatric patients (Patel et al, 2020), video telemedicine for stroke services (Markus \& Brainin, 2020), and remote triage lines for cancer patients in Indonesia (Brahma, 2020).

The NCOVID-19 crisis also brought in the widespread realization that the Internet is an essential utility. Thankfully, most government subsidized cellular plans for the poor users in the US have recently increased free data limits. However, currently, there is no guarantee that everyone has in their possession a smartphone with the basic technical specifications needed for effective consultations with healthcare providers. Another reported challenge is language barriers for the non-native speakers of English. Better integration of the telemedicine technologies with realtime translation software could become handy in such situations. One of the doctors we talked to recommended a patient to communicate with the help of Google Translate, which did not work well. Later, a human translator needed to be found from among the hospital support staff.

The US is definitely a laggard in the deployment of telecommunication technologies for supporting telehealth. To give some contrast, in China, companies like Huawei, ZTE, and China Telecom came together to interconnect the hospital-to-hospital layer of their telemedicine system with $5 G$ technology. In mid-February, Singapore had deployed a GPS tracking system to identify and report those under quarantine. A high-tech giant, South Korea developed a comprehensive trace-test-treat system (See Figure 1 below) including police records, credit card data, transit pass records, CCTV footage and a mobile phone proximity app which alerts passers-by to the presence of nearby patients (Park et al, 2020). 


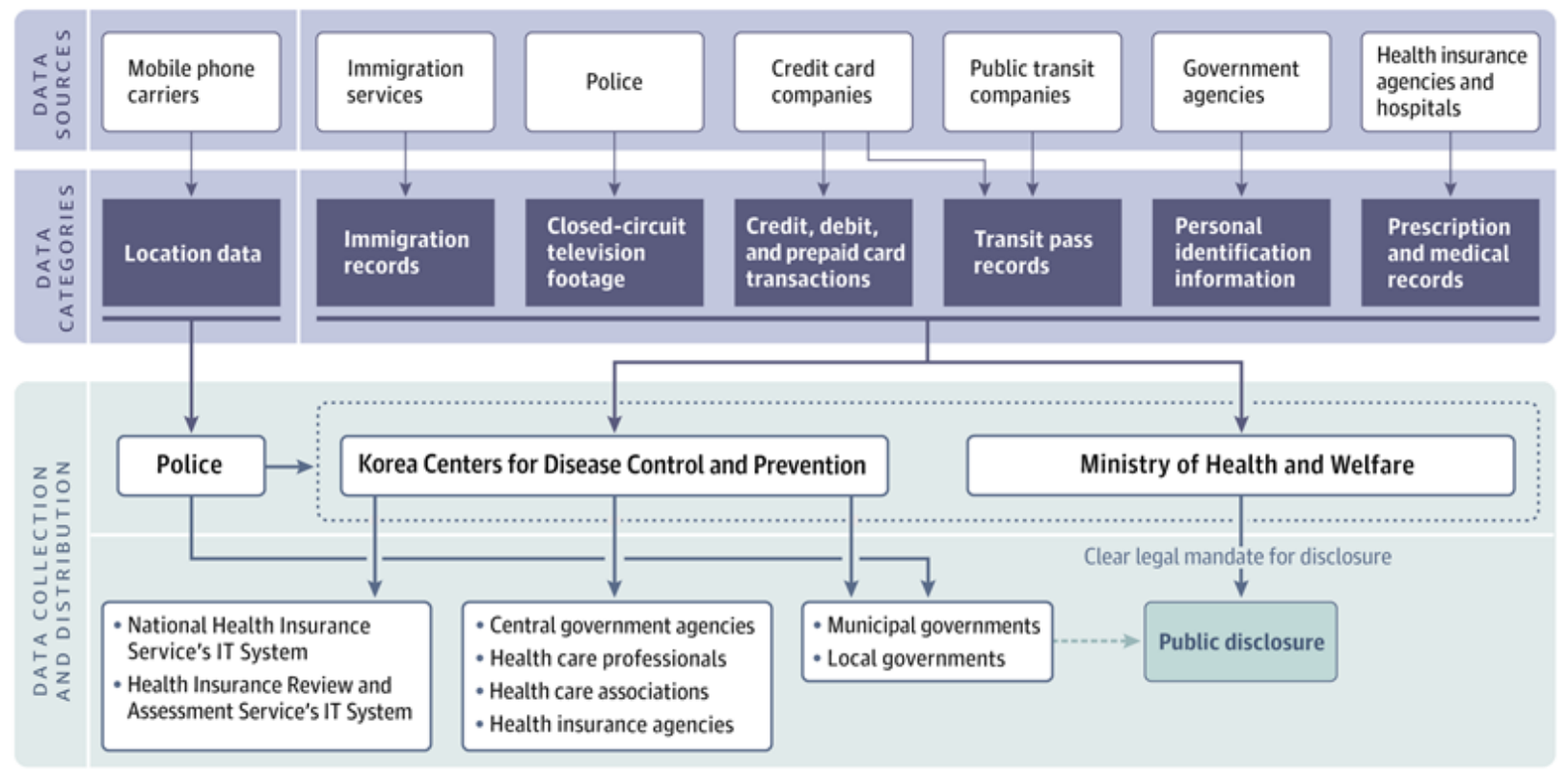

Figure 1 South Korea's Integrated NCOVID-19 System. Source: Park et al, 2020. Retrieved 23rd April, 2020 from https://jamanetwork.com/journals/jama/article-abstract/2765252.

In a similar vein, Taiwan's government made proactive use of big data to cross-reference its national health insurance database with its immigration and custom databases and trigger alerts on a patient's travel history (Lu et al, 2020). In contrast, it took so much more time for Apple and Google to come up with an interoperable contact tracing app in the US, albeit with much less features. It must be noted that the deficiencies in the US system are more related to concerns about privacy and individual liberties rather than the lack of technological knowhow.

The panic created by an unexpected surge for resources in the US also meant patients seeking consultations with remote healthcare providers located abroad. Most patients did this with the knowledge that consultations or purchases made this way would not qualify for insurance benefits. They also seemed to embrace the risk of receiving advise from non-board-certified physicians and getting medicines shipped from unverified sources. We hope, in the post-COVID era, the regulatory system will expand its reach, not to restrain overseas consultations or treatment but to ensure that these players function within the broadly held parameters of the US healthcare system. Such agreements could help with healthcare quality assurance, secure transmission and upkeep of patient records, and bringing down costs.

\section{Future Research Directions}


There are numerous questions about telehealth remaining to be fully answered and more investigations will be needed. For the benefit of future researchers, some of these pressing questions are stated below:

What should be the role of technologies in telehealth (say, as enabler or disrupter of current workflows?). Which are some of the innovative technologies driving the current phase of developments in telehealth? Are technologies developed mainly exogenously and to what extent are healthcare industry experts involved in these processes? What is the role of physician support? Will the increased integration of Al and telemedicine, will general practitioners become redundant in healthcare? How significant a player the social media is?

Will telehealth become a more prominent face of healthcare in the coming years? What are the lingering barriers (e.g. restrictions on hosting and transmitting patient records)? Are human resource management practices in the industry attuned with these developments? What could universities and industry associations do in this regard? What are the ethical implications of increased use of telehealth? Are existing regulatory frameworks conducive to promoting the beneficial aspects of telehealth while at the same time weeding out unethical practices? Where is the accountability and how is it enforced effectively? What are some of the key changes in global consumer behavior that change preferences for telehealth? How have our generally held cultural notions of doctor-patient relationship changed as a result of telehealth? Do telehealth systems give importance to cross-cultural competence? How do stakeholders in the healthcare system such as health insurance companies and pharmaceutical businesses perceive these developments? Has telehealth kept its promise of making healthcare more accessible especially for those who live in remote areas and special populations like the elderly and the immobile ones? What is the relative place of tele-education, teleconsulting, telemonitoring, and telesurgery in the emerging landscape of telehealth?

We need to admit that most of these questions have been addressed in some manner or the other in the extant literature. However, it is equally important to realize that these questions have got dimensions that cannot be captured into any single answer. Differences in organizational and macro-environmental factors would moderate the proposed solutions. 


\section{Conclusion}

The NCOVID-19 crisis threw telehelth into a situation akin to some parents tossing young kids to the ocean expecting them to quickly master swimming. In a typical planned deployment scenario, there would have been numerous rounds of pilot testing and simulations before using it extensively. Although not in anticipation of a pandemic, some countries did these. Norway's "Telemedicine Agder" is one of them: this project, through simulated experiments, studied the implementation of large-scale telemedicine deployments and identified successful service models (Smaradottir, Fensli \& Moe, 2018). Many of the actual uses of telehealth currently are kneejerk reactions that would probably not have passed testing phase. AlDossary, Martin-Khan, Bradford, \& Smith (2017) observe that most telemedicine projects have a poor record of qualifying beyond the trials, based on clinical outcome, economics, or patient satisfaction.

Although we discussed at a great length on technologies, it must be recognized that technology is not the main barrier for further long-term adoption of telehealth. Highlighting the example of the Catalonia region of Spain, Centelles (2020) observes how even a plain old vanilla technology like telephone calling could supplement with traditional consultations and thereby ease the hospital resources for more serious cases. Bottlenecks to telehealth are more about governmental licensing regulations and the attitudes of middlemen such as health insurance companies, particularly in the US scenario.

While public funded healthcare systems in certain countries do not have any motivation to innovate (George \& Salgaonkar, 2006; Mekoth, et al., 2012), a silver line in the misery is that crises like NCOVID-19 are a great exogenous force that can shackle any resistance to change. In the US, some commercial health insurances companies had already agreed to reimburse such remote consultations. Although on a temporary basis, the Centers for Medicare \& Medicaid Services (CMS) in the US has taken an unusually positive stance. The 1135 Waiver issued recently as a result of NCOVID-19 meant hospitals could code these as regular patient visits and get insurance companies to pay for the visits from Medicare as well. However, much more things need to be achieved in this area.

\section{References}


AlDossary, S., Martin-Khan, M. G., Bradford, N. K., \& Smith, A. C. (2017). A systematic review of the methodologies used to evaluate telemedicine service initiatives in hospital facilities. International Journal of Medical Informatics, 97, 171-194.

Azizy, A., Fayaz, M., \& Agirbasli, M. (2020). Do Not Forget Afghanistan in Times of COVID-19: Telemedicine and the Internet of Things to Strengthen Planetary Health Systems. OMICS: A Journal of Integrative Biology.

Brahma, B. (2020). Oncologists and COVID-19 in Indonesia: What can we learn and must do?. Indonesian Journal of Cancer, 14(1), 1-2.

Brandling-Bennett, H. A., Kedar, I., Pallin, D. J., Jacques, G., Gumley, G. J., \& Kvedar, J. C. (2005). Delivering health care in rural Cambodia via store-and-forward telemedicine: a pilot study. Telemedicine Journal \& e-Health, 11(1), 56-62.

Bucatariu, L., \& George, B. (2017). Patient Perception and Choice Factors Related to International Hospitals: A Study in Ho Chi Minh City, Vietnam. Journal of Health \& Medical Informatics, 8(3), 8-277.

Calton, B., Abedini, N., \& Fratkin, M. (2020). Telemedicine in the Time of Coronavirus. Journal of Pain and Symptom Management.

Centelles, E. B. A. (2020). Telemedicine in the face of the COVID-19 pandemic Telemedicine in the face of the COVID-19 pandemic.

Chan, G. H. (2020). A Comparative Analysis of Online, Offline, and Integrated Counseling among Hidden Youth in Hong Kong. Children and Youth Services Review, 105042.

Chauhan, V., Galwankar, S., Arquilla, B., Garg, M., Di Somma, S., El-Menyar, A., ... \& Stawicki, S. P. (2020). Novel coronavirus (COVID-19): Leveraging telemedicine to optimize care while minimizing exposures and viral transmission. Journal of Emergencies, Trauma, and Shock, 13(1), 20.

Deepak, J., Subuhi, K., \& Ishmeet, K. (2020). WhatsApp messenger as a teledermatology tool during coronavirus disease (COVID-19): From Bedside to Phone-side. Clinical and Experimental Dermatology. 
Dinesen, B., Nonnecke, B., Lindeman, D., Toft, E., Kidholm, K., Jethwani, K., ... \& Gutierrez, M. (2016). Personalized telehealth in the future: a global research agenda. Journal of medical Internet research, 18(3), e53.

Gao, Y., Liu, R., Zhou, Q., Wang, X., Huang, L., Shi, Q., ... \& Luo, X. (2020). Application of Telemedicine During the Coronavirus Disease Epidemics: A Rapid Review and MetaAnalysis. medRxiv.

George, B. P., \& Henthorne, T. L. (2009). The incorporation of telemedicine with medical tourism: A study of consequences. Journal of Hospitality Marketing \& Management, 18(5), 512522.

George, B. P., \& Salgaonkar, P. B. (2006). Inter-Relationship Among the Extent of Past Consultations, Recommendation, Satisfaction, and Loyalty in Patient-Doctor Relationship: An Empirical Study. Journal of Global Academy of Marketing Science, 16(3), 17-37.

George, B. P., Henthorne, T. L., \& Williams, A. J. (2010). Determinants of satisfaction and dissatisfaction among preventive and curative medical tourists: a comparative analysis. International Journal of Behavioral and Healthcare Research, 2(1), 5-19.

George, B., \& Paul, J. (2020). Digital Transformation in Business and Society. Springer International Publishing.

Grimes, C. L., Balk, E. M., Crisp, C. C., Antosh, D. D., Murphy, M., Halder, G. E., ... \& Iglesia, C. (2020). A guide for urogynecologic patient care utilizing telemedicine during the COVID-19 pandemic: review of existing evidence. International Urogynecology Journal, 1.

Heinzelmann, P. J., Jacques, G., \& Kvedar, J. C. (2005). Telemedicine by email in remote Cambodia. Journal of telemedicine and telecare, 11(2_suppl), 44-47.

Hollander, J. E., \& Carr, B. G. (2020). Virtually perfect? Telemedicine for COVID-19. New England Journal of Medicine. 
Humphreys, J., Schoenherr, L., Elia, G., Saks, N. T., Brown, C., Barbour, S., \& Pantilat, S. Z. (2020). Rapid Implementation of Inpatient Telepalliative Medicine Consultations during COVID19 Pandemic. Journal of Pain and Symptom Management.

Klein, B. C., \& Busis, N. A. (2020). COVID-19 is catalyzing the adoption of teleneurology. Neurology.

Knopf, A. (2020). Addiction telemedicine comes into its own with COVID-19. Alcoholism \& Drug Abuse Weekly, 32(13), 5-6

Lor, P. H., \& George, B. P. (2014). An appreciative inquiry into the healthcare concerns of the elder Hmong women living in Alaska, USA. Journal of Rural and Community Development, 9(3), 27-41.

Lu, N., Cheng, K. W., Qamar, N., Huang, K. C., \& Johnson, J. A. (2020). Weathering COVID-19 Storm: Successful Control Measures of Five Asian Countries. American Journal of Infection Control.

Markus, H. S. (2020). EXPRESS: COVID-19 and Stroke-A Global World Stroke Organisation perspective. International Journal of Stroke, 1747493020923472.

Mekoth, N., George, B. P., Dalvi, V., Rajanala, N., \& Nizomadinov, K. (2012). Service quality in the public sector hospitals: a study in India. Hospital topics, 90(1), 16-22.

Menon, M., \& George, B. (2018). Social media use for patient empowerment in the Gulf Cooperation Council region. Clinical eHealth, 1(1), 21-27.

Mishra, K. N., \& Chakraborty, C. (2020). A Novel Approach Towards Using Big Data and IoT for Improving the Efficiency of m-Health Systems. In Advanced Computational Intelligence Techniques for Virtual Reality in Healthcare (pp. 123-139). Springer, Cham.

Mohammed, M. N., Syamsudin, H., Al-Zubaidi, S., AK, S., Ramli, R., \& Yusuf, E. (2020). NOVEL COVID-19 DETECTION AND DIAGNOSIS SYSTEM USING IOT BASED SMART HELMET. International Journal of Psychosocial Rehabilitation, 24(7). 
Nagra, M., Vianya-Estopa, M., \& Wolffsohn, J. S. (2020). Could telehealth help eye care practitioners adapt contact lens services during the COVID-19 pandemic?. Contact Lens and Anterior Eye.

Nguyen, T. H., \& Vu, D. C. (2020). The largest epicenter of the coronavirus outbreak in Vietnam. Infection Control and Hospital Epidemiology, 1-2.

Ohannessian, R. (2015). Telemedicine: Potential applications in epidemic situations. European Research in Telemedicine/La Recherche Européenne en Télémédecine, 4(3), 95-98.

Ohannessian, R., Duong, T. A., \& Odone, A. (2020). Global telemedicine implementation and integration within health systems to fight the COVID-19 pandemic: a call to action. JMIR public health and surveillance, 6(2), e18810.

Paraskou, A., \& George, B. P. (2017). The market for reproductive tourism: an analysis with special reference to Greece. Global health research and policy, 2(1), 16.

Paraskou, A., \& George, B. P. (Eds.). (2017). Legal and Economic Considerations Surrounding Reproductive Tourism: Emerging Research and Opportunities: Emerging Research and Opportunities. IGI Global.

Park, S., Choi, G. J., \& Ko, H. (2020). Information Technology-Based Tracing Strategy in Response to COVID-19 in South Korea-Privacy Controversies. JAMA.

Patel, P. D., Cobb, J., Wright, D., Turer, R., Jordan, T., Humphrey, A., ... \& Rosenbloom, S. T. (2020). Rapid Development of Telehealth Capabilities within Pediatric Patient Portal Infrastructure for COVID-19 Care: Barriers, Solutions, Results. Journal of the American Medical Informatics Association.

Portnoy, J., Waller, M., \& Elliott, T. (2020). Telemedicine in the Era of COVID-19. The Journal of Allergy and Clinical Immunology: In Practice. 
Powell, R. E., Henstenburg, J. M., Cooper, G., Hollander, J. E., \& Rising, K. L. (2017). Patient perceptions of telehealth primary care video visits. The Annals of Family Medicine, 15(3), 225-229.

Smaradottir, B. F., Fensli, R., \& Moe, C. E. (2018, June). Recommendations of a laboratory infrastructure for simulation of telemedicine services. In 2018 IEEE Symposium on Computers and Communications (ISCC) (pp. 01217-01220). IEEE.

Smith, A. C., Thomas, E., Snoswell, C. L., Haydon, H., Mehrotra, A., Clemensen, J., \& Caffery, L. J. (2020). Telehealth for global emergencies: Implications for coronavirus disease 2019 (COVID-19). Journal of Telemedicine and Telecare, 1357633X20916567.

Tuckson, R. V., Edmunds, M., \& Hodgkins, M. L. (2017). Telehealth. New England Journal of Medicine, 377(16), 1585-1592.

Wang, J., Qiu, M., \& Guo, B. (2017). Enabling real-time information service on telehealth system over cloud-based big data platform. Journal of Systems Architecture, 72, 69-79.

Zhai, Y., Wang, Y., Zhang, M., Gittell, J. H., Jiang, S., Chen, B., ... \& Wang, X. (2020). From Isolation to Coordination: How Can Telemedicine Help Combat the COVID-19 Outbreak? medRxiv. 